\title{
Signal Transduction Pathways in Breast Cancer: The Important Role of PI3K/Akt/mTOR
}

\author{
Miguel A. Ortega, ${ }^{1,2,3}$ Oscar Fraile-Martínez, ${ }^{1}$ Ángel Asúnsolo, ${ }^{2,4}$ Julia Buján $\mathbb{D}^{1,2}$ \\ Natalio García-Honduvilla $\mathbb{D}^{1,2}$ and Santiago Coca ${ }^{1 D}{ }^{1,2}$ \\ ${ }^{1}$ Department of Medicine and Medical Specialities, Faculty of Medicine and Health Sciences and \\ Networking Biomedical Research Centre on Bioengineering, Biomaterials and Nanomedicine (CIBER-BBN), \\ University of Alcalá, Alcalá de Henares, Madrid, Spain \\ ${ }^{2}$ Ramón y Cajal Institute of Healthcare Research (IRYCIS), Madrid, Spain \\ ${ }^{3}$ Cancer Registry and Pathology Department, Hospital Universitario Principe de Asturias, Alcalá de Henares, Spain \\ ${ }^{4}$ Department of Surgery, Medical and Social Sciences, Faculty of Medicine and Health Sciences, University of Alcalá, \\ Alcalá de Henares, Madrid, Spain
}

Correspondence should be addressed to Santiago Coca; s.coca@uah.es

Received 18 September 2019; Revised 25 November 2019; Accepted 11 January 2020; Published 9 March 2020

Guest Editor: Ludmila Grzybowska-Szatkowska

Copyright ( 2020 Miguel A. Ortega et al. This is an open access article distributed under the Creative Commons Attribution License, which permits unrestricted use, distribution, and reproduction in any medium, provided the original work is properly cited.

\begin{abstract}
Breast cancer is the cancer with the highest prevalence in women and is the number-one cause of cancer mortality worldwide. Cell transduction is a fundamental process in the development and progression of cancer. Modifications in various cell signalling pathways promote tumour cell proliferation, progression, and survival. The PI3K/Akt/mTOR pathway is an example of that, and it is involved in growth, proliferation, survival, motility, metabolism, and immune response regulation. Activation of this pathway is one of the main causes of cancer cell resistance to antitumour therapies. This makes PI3K/Akt/mTOR signalling a crucial object of study for understanding the development and progression of this disease. Thus, this pathway may have a role as a potential therapeutic target, as well as prognostic and diagnostic value, in patients with breast cancer. Despite the existence of selective $\mathrm{PI} 3 \mathrm{~K} / \mathrm{Akt} / \mathrm{mTOR}$ pathway inhibitors and current clinical trials, the cellular mechanisms are not yet known. The present review aims to understand the current state of this important disease and the paths that must be forged.
\end{abstract}

\section{Introduction: Current State of the Disease}

Breast cancer is the most prevalent cancer type in women as well as the leading cause of cancer mortality in this population worldwide, with a peak incidence between 45 and 65 years of age [1]. Although it is not common, breast cancer can also occur in men, with a frequency of 1 in 100 diagnosed cases, representing less than $1 \%$ of all cancers in men [2].

Among the most important risk factors associated with breast cancer are ageing, family history, nulliparity, hormonal factors, such as early menarche or late menopause, and other factors related to lifestyle, such as alcohol consumption, obesity, and physical inactivity $[3,4]$.
Breast cancer can be hereditary or sporadic. The most frequent mutations associated with hereditary cancer include those that affect DNA damage repair (DDR) genes, the most important of which are mutations in the BRCA1, BRCA2, and TP53 genes [5]. Sporadic cancer represents approximately $85 \%$ of all cases of breast cancer and is associated with some of the risk factors mentioned above; however, it has also been associated with exposure to carcinogens, such as air pollutants [6], electromagnetic radiation [7], and DDR gene expression dysregulation [8].

According to their presentation, ductal carcinoma in situ is the most diagnosed breast cancer type, followed by lobular carcinoma in situ [9]. Breast cancer, in turn, is divided into different subtypes based on the presence or absence of the 
estrogen receptor (ER), progesterone receptor (PR), and HER2 receptor. Thus, we can distinguish between a luminal subtype, being ER/PR+, an Her2+ subtype, which has this receptor overexpressed, and a triple negative or basal-like subtype (TNBC). Following this classification, the luminal subtypes can be divided into luminal A, characterized by ER/PR+, HER2-, and low Ki67 expression, and luminal B, characterized by ER/PR+, HER2+, and high Ki67 expression. Subtype Her2+ is ER/PR negative, and the triple negative indicates a lack of all these receptors [10-12].

Cell signal transduction is a fundamental process in the development and progression of cancer. Hanahan and Weinberg [13] noted that tumour cells exhibit a set of characteristics or hallmarks, including uncontrolled proliferation, genomic instability, and apoptosis evasion. To this end, modifications to various cell signalling pathways promote tumour cell proliferation, progression, and survival [14]. These alterations are due to mutations in oncogenes that overexpress certain proteins, mutated proteins that present uncontrolled activity, or inactivation of tumour suppressor genes that favour these processes [15].

Many alterations in breast cancer cells that affect cell signalling pathways have been described. In fact, variations have been described in the responses mediated by calciumsensitive receptors $[16,17]$ or hypoxia-inducible factor [18] or even in the apoptotic cell mechanisms themselves [19]. However, the alterations most studied and most directly involved in the progression and development of breast cancer pathways are those mediated by the ER and human epidermal growth factor type-2 receptors (HER2/Neu or c-ErbB2) [19]. The activity of HER2 receptors in turn promotes the signalling of other pathways such as the mitogen-activated protein kinases (MAPKs) or cell components like glycogen synthase kinase-3 (GSK-3) and PI3K/ Akt/mTOR pathways, both represented in Figure 1, denoting the importance of signal integration and transduction processes in the progression and development of breast cancer [20-23].

\section{Importance of the PI3K/AKT/mTOR Pathway in Cancer}

$\mathrm{PI} 3 \mathrm{~K} / \mathrm{Akt} / \mathrm{mTOR}$ is a cell signalling pathway involved in growth, proliferation, survival, motility, metabolism, and immune response regulation $[24,25]$. This pathway has also been associated with a great variety of diseases and syndromes, such as tuberous sclerosis, Parkinson's disease, and vascular diseases [26-28].

Studies on PI3K/Akt/mTOR have also focused on cancer research. Alterations to this pathway have been found in practically all human tumours, including breast cancer, where up to $60 \%$ of the tumours present different variations that hyperactivate this pathway [29].

The dysregulation of this pathway has been related to a wide variety of cancer hallmarks, including uncontrolled proliferation, genomic instability, and metabolic reprogramming in tumour cells $[13,30]$. In addition, PI3K/Akt/ mTOR pathway activation is one of the main causes of cancer cell resistance to antitumour therapies [31]. This makes the PI3K/Akt/mTOR pathway a crucial object of study for understanding the development and progression of this disease, the role of this pathway as a potential therapeutic target, and the prognostic and diagnostic value of this pathway in patients with breast cancer $[32,33]$.

\section{PI3Ks in Tumours and Existing Therapies}

Phosphoinositide 3-kinases (PI3Ks) are a family of lipid kinases that integrate signals from growth factors, cytokines, and other extracellular stimuli and are related to the cell's response to these kinases [34]. Three different classes of PI3Ks are known, which in turn are divided into different subclasses according to their affinity for certain substrates, their sequence homology, and the functions that each of these classes have in cell signal transduction; class I PI3Ks are the most studied and the most clearly related to oncogenic processes [35]. Class I PI3Ks are divided into the following classes: PI3K IA, activated by receptor tyrosine kinases (RTK) receptors, G-protein-coupled receptors (GPCRs), and oncogenes such as Ras; and PI3K IB, regulated exclusively by GPCRs [36]. Among these, class IA is the most directly implicated in cancer. PI3K class IA consists of a regulatory subunit, which can be $\mathrm{p} 85 \alpha$, p $55 \alpha, \mathrm{p} 50 \alpha, \mathrm{p} 85 \beta$, or $\mathrm{p} 55 \gamma$, and a catalytic subunit, which can be $\mathrm{p} 110 \alpha, \mathrm{p} 110 \beta$, or

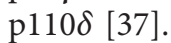

When a cellular receptor is activated, it dimerizes and autophosphorylates in various regions, phosphorylating, in turn, different adapter proteins and being recognized by the p85 subunit, which binds to these phosphorylated residues and generates a conformational change that releases p110, the subunit that exerts catalytic activity. Consequently, a phosphate group is added to phosphatidylinositol 4,5bisphosphate (PIP2), transforming it into phosphatidylinositol (3,4,5)-trisphosphate (PIP3) [37]. Phosphatase and tensin homolog (PTEN) phosphatase, which can remove a phosphate from PIP3 to convert it into PIP2, is the most important negative regulator of this pathway and one of the tumour suppressors with the greatest effect on different types of cancer [38]. PIP3 levels can also be regulated by another tumour suppressor known as inositol polyphosphate-4-phosphatase type II B (INPP4B), which has the same effect as PTEN. INPP4B loss has been considered a marker of aggressiveness in TNBC tumours [39, 40].

Activating mutations in PIK3CA, the gene that encodes the $110 \alpha$ catalytic subunit, have been identified as potent oncogenic mechanisms implicated in the hyperactivation of this pathway (Figure 2); these mutations are especially noteworthy in breast cancer, where up to $27 \%$ of patients have mutations in this gene [41]. Mutations in PI3KCA are more common in luminal A subtype cancers, where they are detected in $45 \%$ of tumours, followed by HER $2+$ mutations with a frequency of 39\%; luminal B represents $30 \%$ of cancers, and TNBC alterations appear in $9 \%$ of cases [24]. These mutations affect mainly the helical domain of the p110 a subunit, reducing its repression by regulatory subunits or facilitating its interaction with IRS1 [42, 43]. PI3K activation is a critical step in oncogenesis and plays a role in treatment resistance in ER+/HER2+ breast cancers. There 


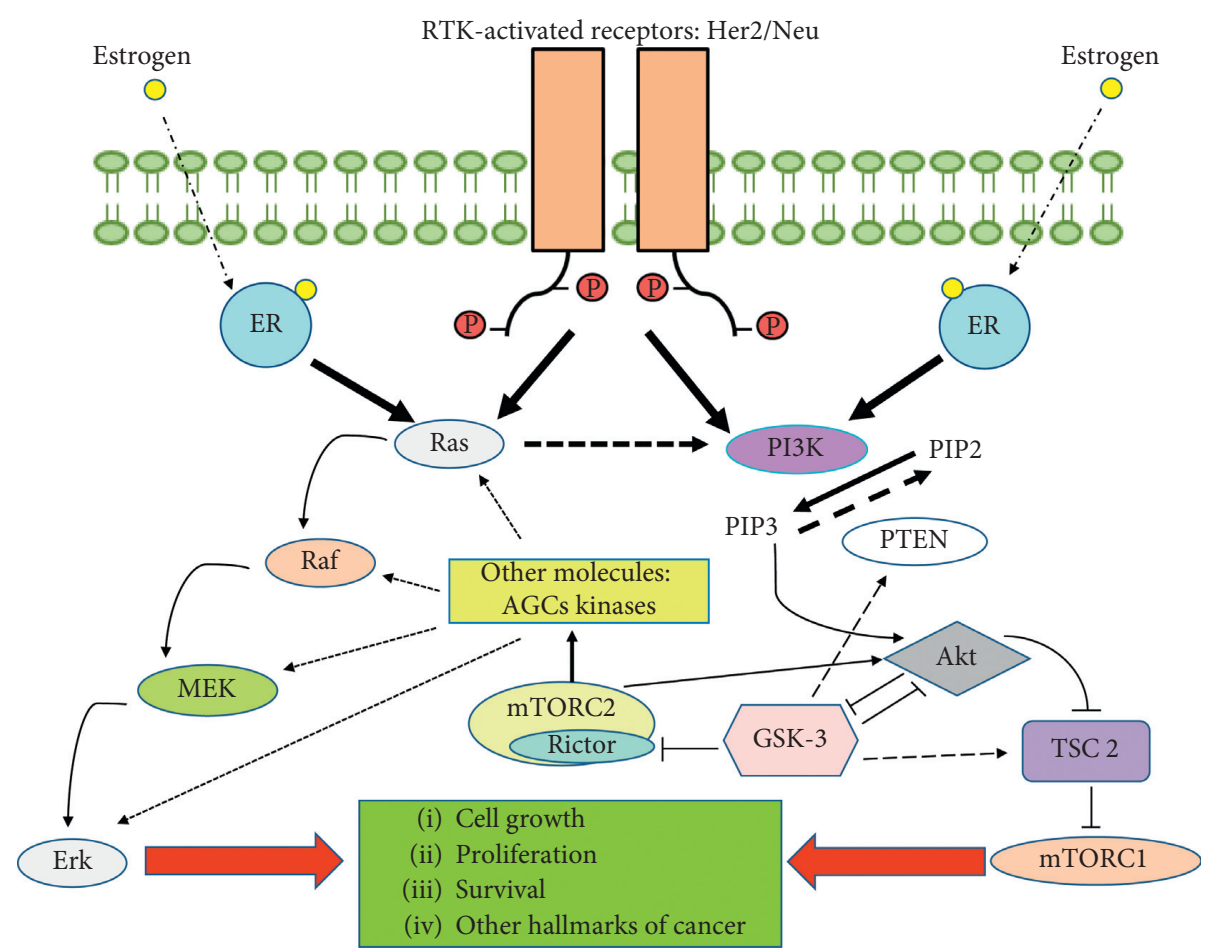

FIGURE 1: Overview of cell signalling mediated by the tyrosine kinase receptors (RTKs) Her2/Neu and estrogen receptors (ERs), two key components of breast cancer development. Their activation initiates the PI3K/Akt/mTOR and MAPK pathways, finally promoting cell growth, proliferation, survival, and other hallmarks of cancer. Although this is a review of PI3K/Akt/mTOR signalling, it is important to understand that the different pathways are connected by different points. In this figure, we have presented two examples: Ras, promoting PI3K activation, and how some AGC kinases (such as SGK-3) activated by mTORC2 also interact with the MAPK pathway. Additionally, GSK-3 plays an important role as well in the regulation of these pathways, represented in the figure. GSK-3 is an example of how complex those interactions are, by the inhibition and activation of different molecules implicated in PI3K and MAPK pathways.

are currently PI3K inhibitors, which are being implemented in clinical trials $[44,45]$.

Mutations in PTEN also result in important alterations in cell signalling in patients with breast cancer. The relationship between the loss of PTEN gene function and unfavourable predictive factors has been observed in various types of cancer, such as gastric, prostate, and colorectal cancer [46-48]. Lu et al. [49] described how the activity exerted by this phosphatase slows cell growth and induces apoptosis and anoikis in breast cancer cells. The results of a meta-analysis of 27 studies and 10,231 cases performed by Li et al. [50] showed that there seems to be an association between PTEN expression loss and tumour aggressiveness in breast cancer patients, especially those with ER, PR, and TNBC tumours, thus demonstrating the involvement of PTEN in the initiation and malignancy of breast cancer tumour cells.

There are multiple PI3K inhibitors that have been developed or are in the study phase. These are classified according to their specificity for each isoform and can be divided into (1) first-generation inhibitors, also known as pan-inhibitors of PI3K, which target all the different PI3K class I isoforms $(\alpha, \beta, \delta$, or $\gamma)$, (2) second-generation inhibitors, which are specific to any of these isoforms, or (3) dual PI3K/mTOR inhibitors. However, the efficacy shown by these types of inhibitors as therapeutic agents is far from that expected due to the coexistence of various mutations present in tumour cells, compensatory feedback cycles, or the toxicity associated with these treatments [24]. That is why research has focused on these inhibitors in combination with other drugs, although much work remains.

Of the first-generation inhibitors, breast cancer studies have focused on the use of pictilisib and buparlisib. The role of pictilisib as a pan-inhibitor (GDC-0941) in the inhibition of the metastatic phenotype in thyroid carcinomas has been demonstrated due to its action on PI3K and HIF-1 $\alpha$ [51]. Zou et al. [52] observed the synergistic role that this drug had with the MEK inhibitor UO126 in inhibiting NSCLC cell growth. A study conducted by Schmid et al. [53] showed how the combination of pictilisib with anastrozole obtained a better response in inhibiting breast cancer cell proliferation. However, in a study conducted by Krop et al. [54], the combined use of pictilisib with fulvestrant was not associated with an improvement in the treatment of patients with advanced-stage breast cancer resistant to endocrine therapy, regardless of the presence or absence of PI3KCA mutations, measured in terms of progression-free survival (PFS).

Buparlisib (BKM120), another pan-inhibitor of PI3K, has demonstrated synergistic growth inhibitory effects in combination with agents targeting the HER2 receptor in preclinical studies [45]. Several early clinical trials have shown positive results for the use of buparlisib in breast cancer patients [55-57]. Another phase II clinical trial developed by Loibl et al. [58] studied the combination of 


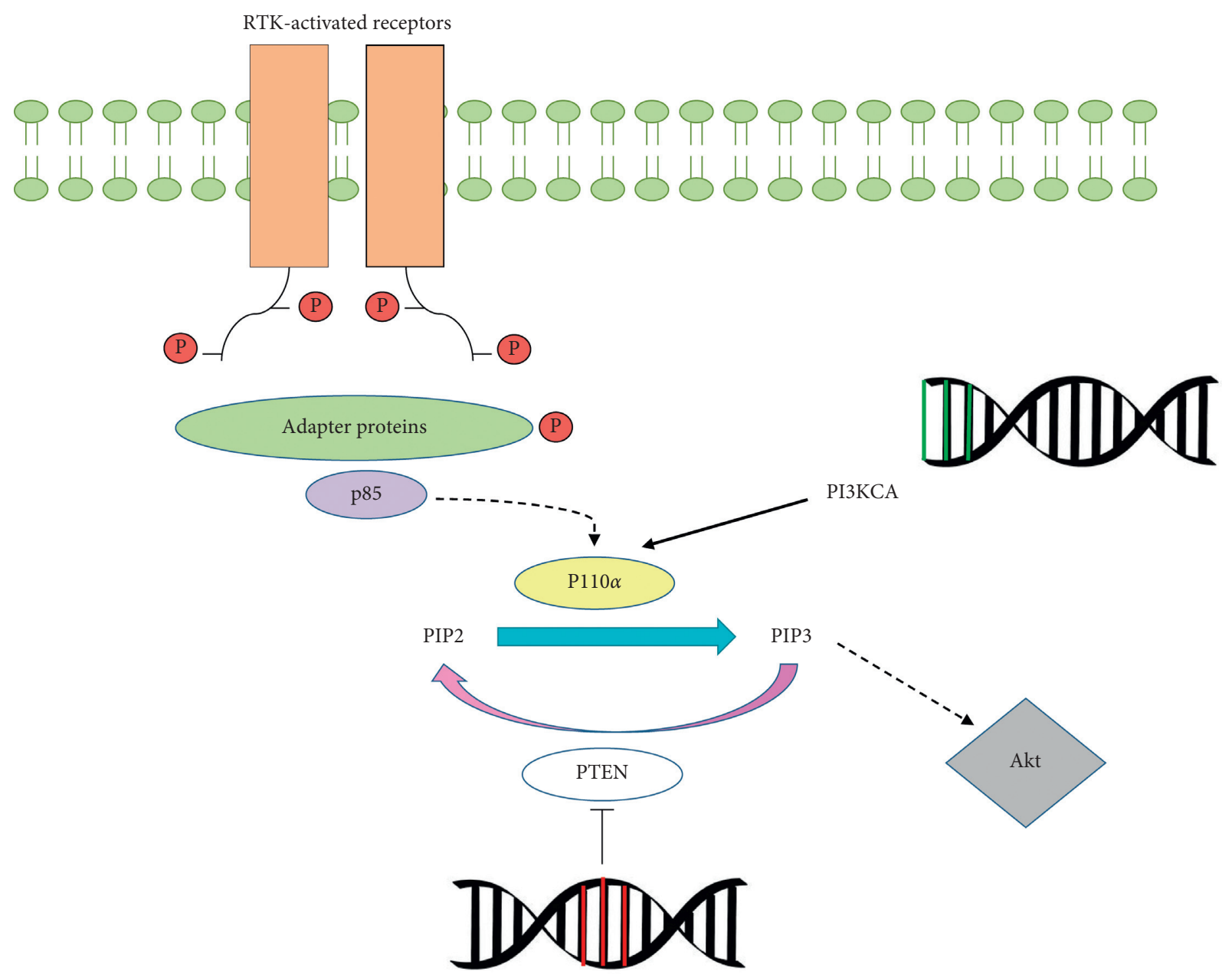

FIGURE 2: Mechanism of action of PI3K and PTEN. RTK receptors are activated, phosphorylating themselves and other series of adapter proteins, such as IRS-1. In this case, the regulatory subunit p 85 binds to these residues and releases the catalytic subunit p $110 \alpha$, which adds a phosphate to PIP2 and transforms it into PIP3, which will subsequently activate Akt. PTEN prevents this activation by dephosphorylating PIP3. The loss of function of this gene, represented in red, or activating PI3KCA mutations, shown in green, can overactivate this route, favouring the development of cancer.

buparlisib with trastuzumab and paclitaxel in women with primary HER2+ tumours. The results showed little reliability in its use; however, high rates of objective response and a reduction in the Ki67 cell proliferation marker in the ER+HER2+ subgroup denote the importance of future investigations of therapies targeting this pathway. Furthermore, two important clinical trials need to be mentioned here: BELLE-2 and BELLE-3. Both were double-blind, randomized, placebo-controlled phase 3 trials in which the use of buparlisib was analysed in hormone receptor-positive Her2-negative postmenopausal women. BELLE-2 aimed to evaluate the combination of buparlisib plus fulvestrant versus placebo and fulvestrant. The results showed that the use of this PI3K inhibitor combined with endocrine therapy was associated with a significant improvement compared to fulvestrant alone. However, this combination was also found to have considerable toxicity, limiting its efficacy [59]. In BELLE-3, 432 postmenopausal women who had previously received endocrine therapy and mTOR inhibitors were divided again into two groups: a group that received fulvestrant and buparlisib and a control group that received only fulvestrant. Patients with fulvestrant plus buparlisib had a longer PFS than those who received fulvestrant alone, but again, the toxicity of this combination restricts its use [60]. On the whole, these works highlight the importance of performing further studies with $\mathrm{PI} 3 \mathrm{~K}-\alpha$-specific inhibitors to provide the greatest benefits.

Currently, research is also aimed at the use of secondgeneration PI3K inhibitors, such as alpelisib (BYL719) and taselisib (GDC0032), which target the PI3K- $\alpha$ isoform, as a higher-safety profile is expected compared to previous inhibitors [61]. Numerous previous studies seem to indicate that mutations in PI3KCA in ER + tumours decrease the response rate of breast cancer tumours to antiestrogenic therapy $[62,63]$. A series of preclinical cell models developed by Fritsch et al. [64] demonstrated how cells with mutations in PIK3CA had greater sensitivity to alpelisib, while cell lines without this mutation had selective sensitivity. A phase III clinical trial showed how patients with advanced tumours and mutations in PIK3CA benefited from the use of alpelisib in combination with fulvestrant; compared with fulvestrant with placebo, this combination increased the PFS and 
response rates [65]. In fact, alpelisib has recently become the first PI3K inhibitor approved by the FDA for the treatment of breast cancer, so the study of this type of inhibitor is a growing point of interest in actual research. Other preclinical studies also reported the efficacy of taselisib in cellular models with mutations in PIK3CA in breast cancer [66]. SANDPIPER is an ongoing randomized, double-blind phase III clinical trial with 631 postmenopausal women with ER + HER2- PI3KCA mutations who showed progression or recurrence during or after aromatase inhibitor (AI) therapy. This trial aims to investigate the efficacy and safety of taselisib plus fulvestrant in comparison to a fulvestrant alone control group (NCT02340221). SOLAR-1 is another ongoing phase III clinical study designed to evaluate the use of alpelisib plus fulvestrant in ER/PR + Her2- advanced cancer patients after treatment with AI (NCT02437318). Currently, GDC-0077, another second-generation PI3K- $\alpha$ inhibitor, is being tested in a phase I clinical trial in patients with PIK3CA mutant solid tumours that are locally advanced or metastatic, including breast cancer, and GDC-0077 is being tested in combination with targeted therapies focused on the ER/PR+Her2- subtype (NCT03006172).

Regarding third-generation inhibitors or dual PI3K/ mTOR inhibitors, the role of some of these inhibitors, such as BEZ235, has been studied in combination with other inhibitors, such as everolimus (RAD-001), and these inhibitors have been shown to exert a synergistic action by decreasing the growth of some TNBC cell lines, such as MDA-MB-231 cells or the ER + MCF-7 line [67]. The combination of BEZ235 with autophagy inhibitors has also been studied, and these combinations have resulted in inhibited proliferation and increased apoptosis in breast cancer cells [68]. However, clinical studies of BEZ-235 have not yielded promising outcomes. A phase I/IB study of BEZ-235 in patients with Her2+ advanced breast cancer showed that the safety profile of this inhibitor was not adequate [69]. Nevertheless, clinical studies of BEZ235 are ongoing in patients with metastatic breast cancer [70]. Some previous studies indicate how AIs can be combined with PI3K/mTOR inhibitors for treating patients with resistance to endocrine therapy who present with ER+HER-metastatic cells. In addition, it has been studied how the PF-04691502 inhibitor is currently used for combined treatment with tamoxifen for treating breast cancer stem cells (BCSCs); in this manner, the resistance they presented when tamoxifen was applied in the absence of another drug was avoided, and the importance of this pathway in therapy resistance and in the formation of mammals was shown [71]. Finally, another dual PI3K/mTOR inhibitor, GDC-0980, has been shown to be particularly effective in BRCA-competent TNBC when combined with a PARP inhibitor and carboplatin because it inhibits the DDR system [72]. Studies on the efficacy of DDR inhibitors in breast cancer therapy are discussed in previous reviews [73].

\section{AKT in Tumours and Existing Therapies}

RAC-alpha serine/threonine-protein kinase (Akt), also known as protein kinase $\mathrm{B}(\mathrm{PKB})$, is a serine-threonine kinase that has three different isoforms encoded by three different genes: Akt1, Akt2, and Akt3. However, it is Akt1 that has been most associated with cancer [74]. Akt1 binds to PIP3 via pleckstrin homology $(\mathrm{PH})$ domains [75]. This interaction localizes Akt to the cell membrane, resulting in the subsequent phosphorylation of the Thr308 and Ser473 residues by 3-phosphoinositide-dependent protein kinase-1 and -2 (PDK1 and PDK2). While PDK1 is activated by PIP3, PDK2 forms part of the mTORC2 complex [76].

The effects of Akt activation include the activation of MDM2, a p53 inhibitor, and the inhibition of other proteins involved in stimulating cell survival in stress situations [77]. The activation of Akt has also been associated with the inhibition of p21 and p27, which are involved directly in cell cycle control [78], and GSK-3 $\beta$, as shown in Figure 3. GSK$3 \beta$ inactivation is directly related to cell metabolism reprogramming and, more specifically, to the uptake and use of glucose, which favours the Warburg effect [79]. However, the role of GSK- $3 \beta$ in cancer remains controversial. Some studies have reported that GSK-3 $\beta$ may function as a tumour promoter. GSK- $3 \beta$ knockdown cells were associated with decreased levels of Bcl-2 and VEGF, thus suggesting the role of GSK-3 $\beta$ in inhibiting apoptosis and promoting angiogenesis [80]. On the other hand, previous findings have indicated GSK- $3 \beta$ as a tumour suppressor in breast cancer because it increases the sensitivity of chemotherapy and inhibits PI3K/Akt and Wnt signalling, thus playing a key role in the cell cycle and survival $[81,82]$. The interaction of GSK-3 with PI3K/Akt pathway is represented in Figure 1. The activation of Akt is also related to the activation of mTORC1. In addition, Akt is involved in the activation and inactivation of various transcription factors; for example, it binds 14-3-3 proteins and prevents the translocation of FOXO to the nucleus [83]. Akt is also important in the regulation of NF- $\kappa \beta$-dependent gene transcription and CREB-1 overactivation, thus promoting the expression of antiapoptotic genes such as Bcl-2 and mcl-1 [84, 85].

The hyperactivation of Akt and consequently mTOR may facilitate the resistance that some patients with breast cancer have to endocrine therapies; in these patients, an inverse correlation was established between Akt activation and a partial treatment response [86, 87].

In breast cancer, the most frequent mutation is E17KAkt1, representing $2 \%$ of all breast cancers. This mutation increases the affinity of the $\mathrm{PH}$ domain for lipids, resulting in the constitutive localization of Akt in the cell membrane [88]. Currently, there are phase I and II clinical trials with allosteric and catalytic Akt inhibitors. Hyman et al. [89] proposed the use of the E17K-Akt1 mutation as a predictive marker of the response to the catalytic inhibitor AZD5363, with promising results. However, studies with the allosteric inhibitor MK-2206 combined with hormone therapy reported benefit ratios of only $42 \%$ [57].

In addition, recent studies indicate that there are other proteins also activated by PI $3 \mathrm{~K}$ that contribute to the development of cancer. Lien et al. [30] described that Akt is not always hyperactivated in the presence of PI3KCA or PTEN mutations; the authors also showed the role that other PI3Kdependent proteins may play in the resistance to pathway inhibitors and how these proteins can substitute for Akt- 


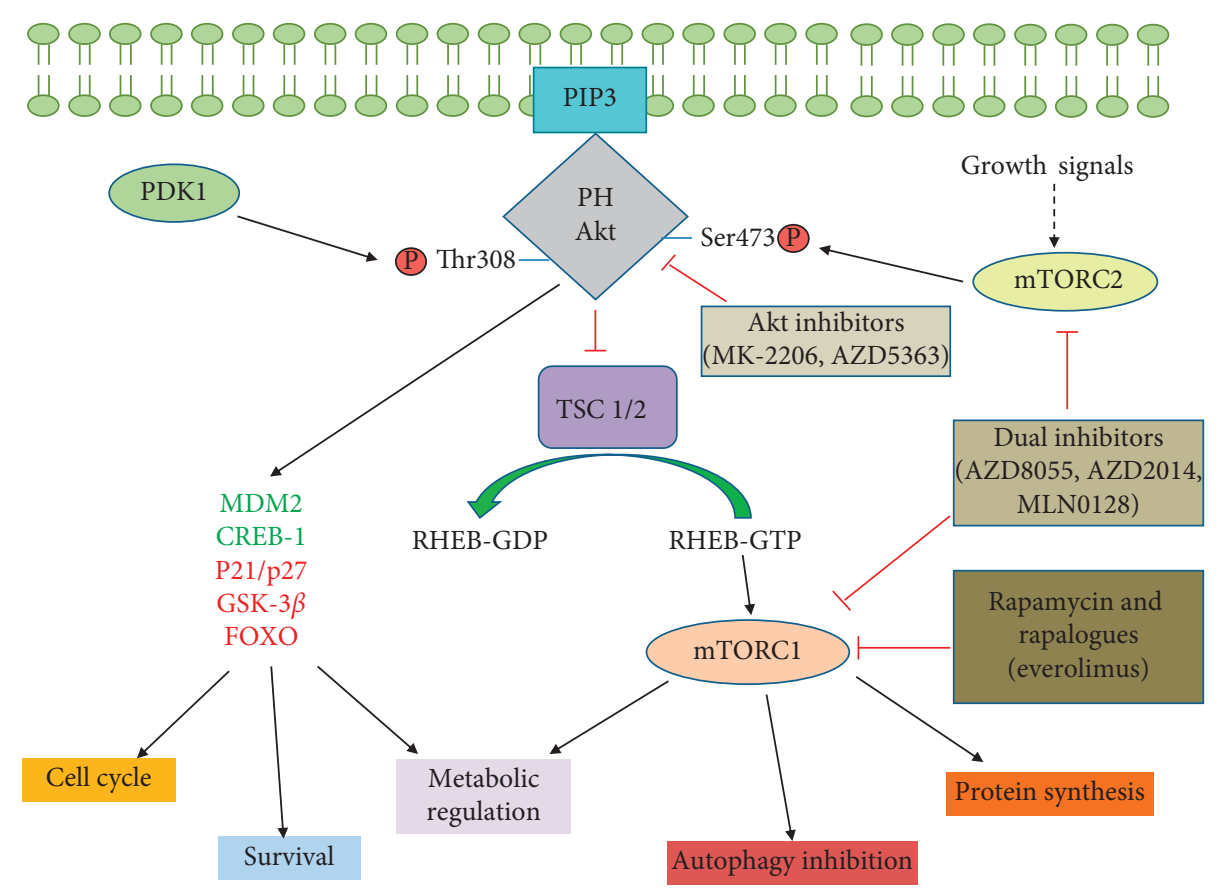

Figure 3: Activation of Akt after joining to PIP3 by its PH domain. PDK1 and PDK2, present in mTORC2, phosphorylate Akt, which will activate and inhibit a series of genes, transcription factors, and proteins, represented in red, such as the TSC complex, which will eventually activate mTORC1, resulting in a series of cellular responses. We have remarked in green the components which are stimulated or overactive in these cells. This figure also shows the main therapies developed to focus on this pathway.

mediated signalling, indicating that further knowledge regarding these pathways is important for developing promising future therapies (Figure 3).

\section{5. mTOR in Tumours and Existing Therapies}

The mammalian target of rapamycin (mTOR) is a protein kinase comprising two distinct complexes, mTORC1 and mTORC2. Both complexes are associated with a set of proteins that can be common or specific to each complex [90]. mTORC1 is a complex formed by the mTOR, Raptor, Deptor, Pras40, and mLST8 proteins [91]. This complex is activated by Akt via inhibition of the tuberous sclerosis complex (TSC) [92]. In turn, TSC inhibits mTORC1 due to its ability to inactivate RHEB, a GTPase that activates this complex. mTORC1 responds to amino acids, stress, oxygen levels, energy needs, and growth signals [24]. The activation of mTORC1 seems to be involved in multiple cellular processes, including protein synthesis control, through phosphorylation of the ribosomal proteins $\mathrm{S} 6 \mathrm{~K} 1$ and $4 \mathrm{E}-$ $\mathrm{BP} 1$, the regulation of metabolism, and the inhibition of autophagy [93].

mTORC2 is a complex that shares certain components with mTORC1, such as mTOR kinase, mLST8, and Deptor. In addition, it contains the Rictor protein and the subunits Sin 1 and Protor 1/2 [91]. This complex is insensitive to rapamycin, unlike the mTORC1 complex [94]. mTORC2 is activated by growth signals and controls cell proliferation and survival processes, as well as the cytoskeleton, mainly by phosphorylating other proteins such as Akt, which, in turn, is the main activator of mTORC1 [95]. In fact, mTORC2 phosphorylates AGC kinase family members, which are Akt, SGK, and PKC (Figure 1), all of which have oncogenic effects $[96,97]$. The role of SGKs is also interesting in cancer because they interact at various levels with MAPK signalling and are related to the tumourigenesis process in PI3K mutants through Akt-independent but SGK-3-dependent malignant transformation (Figure 1) [98]. mTORC2 also interacts with the retinoblastoma $(\mathrm{Rb})$ tumour suppressor. Previous studies have demonstrated that $\mathrm{Rb}$ may inhibit Akt activation by inhibiting the mTORC2/PDK1 phosphorylation of Ser-473 Akt [99]. Furthermore, mTORC2 has been associated with the control of the expression of glycolytic genes by epigenetic regulation of $\mathrm{H} 3 \mathrm{~K} 56 \mathrm{Ac}$ levels in glioma cells [100], suggesting the different and important roles that this complex may have in cancer progression and regulation.

Everolimus is one of the main drugs that targets mTOR, and numerous studies have demonstrated its effectiveness in breast cancer therapy [101]. Du et al. [102] reported the efficacy of everolimus in the inhibition of antiapoptotic proteins such as Bcl-2, as well as in breast cancer cell cycle and growth arrest and in disease progression. Other studies have shown the efficacy of everolimus in the treatment of advanced ER + PR + breast cancer [103]. Everolimus is an allosteric inhibitor of mTORC1 but not mTORC2 and can increase Akt phosphorylation by not binding the latter inhibited complex. Dual inhibitors of mTOR, affecting both complexes, have been studied. Leung et al. [104] demonstrated in vitro the synergy that this drug presented with other pathway inhibitors, such as dual mTOR inhibitors, representing new strides for greater efficacy of the developed therapies targeting this pathway. 
Among the dual inhibitors are AZD8055, AZD2014, and MLN0128, which are in clinical development but have already demonstrated efficacy in numerous studies $[105,106]$. These inhibitors show better results compared to mTORC1 inhibitors in blocking the PI3K/Akt/mTOR pathway as measured by 4-EBP1, SK6, and p-Akt inhibition [107]. The effectiveness of these inhibitors has been demonstrated even in everolimus resistance acquired by mutations in the rapamycin binding domain of mTOR (Figure 3) [108]. Here, it is important to discuss the role of mTOR inhibitors and their relationship with the DNA-dependent protein kinase catalytic subunit (DNA-PKcs), another key component of the DDR system. Low levels of DNA-PKcs protein expression were related to higher tumour grade, dedifferentiation and mitotic index, and poor survival $[73,109]$. CC115, a dual inhibitor of mTOR and DNA-PKcs, has been shown to inhibit cell growth in vitro by blocking DDR pathways, thus inducing apoptosis in many cancer lines, including breast cancer cells [110]. Currently, a phase I clinical trial with CC-115 is ongoing. Other studies also report the role of the DNA-PK inhibitor NU7441 in sensitizing breast cancer cells to ionizing radiation and doxorubicin [111].

Recently, an mTORC2-selective inhibitor based on nanotechnology for HER2 amplification combined with the HER2 inhibitor lapatinib was validated in TNBC, showing its promising efficacy for both subtypes [112].

\section{IRS4 in Tumours and the Relationship with PI3K/Akt/mTOR in Breast Cancer}

Insulin receptor substrates (IRSs) are a set of adaptive cytoplasmic proteins that were originally identified by their role in insulin signalling [113]. The IRS family comprises six members. IRS1 and IRS2 are the best studied variants due to their broad expression in different tissues, and they strongly resemble each other in their structures [114]. IRS3 has been found in rodents but not in humans [115]. IRS5/DOK4 and IRS6/DOK5 are two distant members of the family with greater resemblance to each other than to the other IRSs [116]. The present study will review IRS4, which has been implicated in some types of cancer, such as breast cancer, squamous carcinoma of the lung, sarcomas, and acute lymphoblastic leukaemia $[117,118]$.

IRS phosphorylation occurs at tyrosine residues, generally through RTK-type receptors, thus inducing a signalling cascade that, in turn, promotes the activation of other pathways such as PI3K/Akt or MAPK (Figure 1) [114, 119]. However, Ikink et al. [120] demonstrated that IRS4, unlike other family members, did not require growth factors, leading to the proliferation of mammary epithelial cells due to elevated basal activation of the PI3K/Akt/mTOR pathway. This activation is achieved through the interaction of IRS4 with the p85 subunit [121]. In addition, an association was found between adenovirus infection or certain retroviruses and increased levels of IRS4 [121, 122].

Other studies have shown that there is a positive correlation between IRS4 levels and other proteins involved in breast cancer, such as Breast Tumor Kinase (Brk), a kinase that is overexpressed in $80 \%$ of breast tumour cells, indicating the importance of this protein in the development of breast cancer $[123,124]$.

It has also been observed that IRS4 induces resistance to trastuzumab and lapatinib, two treatments directed against HER 2+ breast cancer cells, because it is involved directly in the hyperactivation of the $\mathrm{PI} 3 \mathrm{~K} / \mathrm{Akt} / \mathrm{mTOR}$ pathway, which is ultimately responsible for this resistance, as shown in previous studies; thus, IRS4 is a promising therapeutic target for these types of tumours that are resistant to treatment $[125,126]$.

\section{Future Directions}

The study of cellular events that occur in oncogenic processes is essential to understand the treatment actions we can take. Furthermore, it is important to understand that carcinogenesis involves a wide range of changes in tumour cells that enable their malignant transformation. From this perspective, the PI3K/Akt/mTOR cell signalling pathway is one of the key points of study that could result in improvements in the existing survival curves after good anatomopathological studies and analyses of the efficacy and efficiency of the inhibition. Despite the existence of selective $\mathrm{PI} 3 \mathrm{~K} / \mathrm{Akt} / \mathrm{mTOR}$ inhibitors and current clinical trials, the underlying cellular mechanisms are not yet known. Studying the existing scientific literature helps to understand the current state and the possible paths to be taken. To maximize the efficacy of the inhibitors and decrease their toxicity, $\mathrm{PI} 3 \mathrm{~K} / \mathrm{Akt} / \mathrm{mTOR}$ targeting is commonly combined with additional treatments, such as endocrine therapy or DDR inhibitors. Current challenges may be to minimize the side effects of these therapies as well as to increase their specificity, thus bringing more benefits to the patients who need to receive this type of treatment.

\section{Conflicts of Interest}

The authors declare that they have no conflicts of interest regarding the publication of this paper.

\section{Authors' Contributions}

Miguel A. Ortega and Oscar Fraile-Martínez contributed equally to this work. Natalio García-Honduvilla and Santiago Coca share senior authorship of this work.

\section{Acknowledgments}

This work was supported by grants from the B2017/BMD3804 MITIC-CM (Community of Madrid, Spain), cofinanced by the European Development Regional Fund "A Way to Achieve Europe" (ERDF).

\section{References}

[1] J. Ferlay, I. Soerjomataram, R. Dikshit et al., "Cancer incidence and mortality worldwide: sources, methods and major patterns in GLOBOCAN 2012," International Journal of Cancer, vol. 136, no. 5, pp. E359-E386, 2015. 
[2] M. Elbachiri, S. Fatima, Z. Bouchbika et al., "Breast cancer in men: about 40 cases and literature review," Pan African Medical Journal, vol. 28, p. 287, 2017.

[3] J. Kruk and H. Aboul-Enein, "Environmental exposure, and other behavioral risk factors in breast cancer," Current Cancer Therapy Reviews, vol. 2, no. 1, pp. 3-21, 2006.

[4] A. Howell, A. S. Anderson, R. B. Clarke et al., "Risk determination and prevention of breast cancer," Breast Cancer Research, vol. 16, no. 5, p. 446, 2014.

[5] L. Melchor and J. Benítez, "The complex genetic landscape of familial breast cancer," Human Genetics, vol. 132, no. 8, pp. 845-863, 2013.

[6] Y. Wei, J. Davis, and W. F. Bina, "Ambient air pollution is associated with the increased incidence of breast cancer in US," International Journal of Environmental Health Research, vol. 22, no. 1, pp. 12-21, 2012.

[7] M. Nazıroğlu, S. Tokat, and S. Demirci, "Role of melatonin on electromagnetic radiation-induced oxidative stress and $\mathrm{Ca}^{2+}$ signaling molecular pathways in breast cancer," Journal of Receptors and Signal Transduction, vol. 32, no. 6, pp. 290-297, 2012.

[8] S. Ingvarsson, B. I. Sigbjornsdottir, C. Huiping et al., "Mutation analysis of the CHK2 gene in breast carcinoma and other cancers," Breast Cancer Research, vol. 4, no. 3, p. R4, 2002.

[9] E. M. Ward, C. E. DeSantis, C. C. Lin et al., "Cancer statistics: breast cancer in situ," CA: A Cancer Journal for Clinicians, vol. 65, no. 6, pp. 481-495, 2015.

[10] M. Abubakar, J. Figueroa, H. R. Ali et al., "Combined quantitative measures of ER, PR, HER2, and KI67 provide more prognostic information than categorical combinations in luminal breast cancer," Modern Pathology, vol. 32, no. 9, pp. 1244-1256, 2019.

[11] T. Sorlie, C. M. Perou, R. Tibshirani et al., "Gene expression patterns of breast carcinomas distinguish tumor subclasses with clinical implications," Proceedings of the National Academy of Sciences, vol. 98, no. 19, pp. 10869-10874, 2001.

[12] S. J. Schnitt, "Will molecular classification replace traditional breast pathology?" Int J Surg Pathol, vol. 18, no. 3 Suppl, pp. 162S-166S, 2010.

[13] D. Hanahan and R. A. Weinberg, "Hallmarks of cancer: the next generation,” Cell, vol. 144, no. 5, pp. 646-674, 2011.

[14] M. A. Feitelson, A. Arzumanyan, R. J. Kulathinal et al., "Sustained proliferation in cancer: mechanisms and novel therapeutic targets," Seminars in Cancer Biology, vol. 35, no. Suppl, pp. S25-S54, 2015.

[15] R. Sever and J. S. Brugge, "Signal transduction in cancer," Cold Spring Harbor Perspectives in Medicine, vol. 5, no. 4, Article ID a006098, 2015.

[16] X. Li, X. Kong, L. Jiang et al., "A genetic polymorphism (rs17251221) in the calcium-sensing receptor is associated with breast cancer susceptibility and prognosis," Cellular Physiology and Biochemistry, vol. 33, no. 1, pp. 165-172, 2014.

[17] W. Kim, F. M. Takyar, K. Swan et al., "Calcium-Sensing receptor promotes breast cancer by stimulating intracrine actions of parathyroid hormone-related protein," Cancer Research, vol. 76, no. 18, pp. 5348-5360, 2016.

[18] N. Kozlova, M. Wottawa, D. M. Katschinski, G. Kristiansen, and T. Kietzmann, "Hypoxia-inducible factor prolyl hydroxylase 2 (PHD2) is a direct regulator of epidermal growth factor receptor (EGFR) signaling in breast cancer," Oncotarget, vol. 8, no. 6, pp. 9885-9898, 2017.

[19] E. P. Booy, E. S. Henson, and S. B. Gibson, "Epidermal growth factor regulates Mcl-1 expression through the
MAPK-Elk-1 signalling pathway contributing to cell survival in breast cancer," Oncogene, vol. 30, no. 20, pp. 2367-2378, 2011.

[20] Y. Feng, M. Spezia, S. Huang et al., "Breast cancer development and progression: risk factors, cancer stem cells, signaling pathways, genomics, and molecular pathogenesis," Genes \& Diseases, vol. 5, no. 2, pp. 77-106, 2018.

[21] A. W. Burgess, "EGFR family: structure physiology signalling and therapeutic targets," Growth Factors, vol. 26, no. 5, pp. 263-274, 2008.

[22] M. A. Ortega, Á. Asúnsolo, B. Romero et al., "Unravelling the role of MAPKs (ERK1/2) in venous reflux in patients with chronic venous disorder," Cells Tissues Organs, vol. 206, no. 4-5, pp. 272-282, 2018.

[23] M. A. Hermida, J. Dinesh Kumar, and N. R. Leslie, "GSK3 and its interactions with the PI3K/AKT/mTOR signalling network," Advances in Biological Regulation, vol. 65, pp. 515, 2017.

[24] A. Guerrero-Zotano, I. A. Mayer, and C. L. Arteaga, "PI3K/ $\mathrm{AKT} / \mathrm{mTOR}$ : role in breast cancer progression, drug resistance, and treatment," Cancer and Metastasis Reviews, vol. 35, no. 4, pp. 515-524, 2016.

[25] D. A. Fruman, H. Chiu, B. D. Hopkins, S. Bagrodia, L. C. Cantley, and R. T. Abraham, "The PI3K pathway in human disease," Cell, vol. 170, no. 4, pp. 605-635, 2017.

[26] K. Switon, K. Kotulska, A. Janusz-Kaminska, J. Zmorzynska, and J. Jaworski, "Tuberous sclerosis complex: from molecular biology to novel therapeutic approaches," IUBMB Life, vol. 68 , no. 12 , pp. 955-962, 2016.

[27] D. D. Feng, W. Cai, and X. Chen, "The associations between Parkinson's disease and cancer: the plot thickens," Translational Neurodegeneration, vol. 4, no. 1, p. 20, 2015.

[28] M. A. Ortega, Á. Asúnsolo, J. Leal et al., "Implication of the $\mathrm{PI} 3 \mathrm{~K} / \mathrm{Akt} / \mathrm{mTOR}$ pathway in the process of incompetent valves in patients with chronic venous insufficiency and the relationship with aging," Oxidative Medicine and Cellular Longevity, vol. 2018, Article ID 1495170, 14 pages, 2018.

[29] J. A. Engelman, "Targeting PI3K signalling in cancer: opportunities, challenges and limitations," Nature Reviews Cancer, vol. 9, no. 8, pp. 550-562, 2009.

[30] E. C. Lien, C. C. Dibble, and A. Toker, "PI3K signaling in cancer: beyond AKT," Current Opinion in Cell Biology, vol. 45 , pp. 62-71, 2017.

[31] M. Martini, M. C. De Santis, L. Braccini, F. Gulluni, and E. Hirsch, "PI3K/AKT signaling pathway and cancer: an updated review," Annals of Medicine, vol. 46, no. 6, pp. 372-383, 2014.

[32] D. A. Fruman and C. Rommel, "PI3K and cancer: lessons, challenges and opportunities," Nature Reviews Drug Discovery, vol. 13, no. 2, pp. 140-156, 2014.

[33] N. M. Davis, M. Sokolosky, K. Stadelman et al., "Deregulation of the EGFR/PI3K/PTEN/Akt/mTORC1 pathway in breast cancer: possibilities for therapeutic intervention," Oncotarget, vol. 5, no. 13, pp. 4603-4650, 2014.

[34] L. M. Thorpe, H. Yuzugullu, and J. J. Zhao, "PI3K in cancer: divergent roles of isoforms, modes of activation and therapeutic targeting," Nature Reviews Cancer, vol. 15, no. 1, pp. 7-24, 2015.

[35] J. A. Engelman, J. Luo, and L. C. Cantley, “The evolution of phosphatidylinositol 3-kinases as regulators of growth and metabolism," Nature Reviews Genetics, vol. 7, no. 8, pp. 606-619, 2006. 
[36] P. Liu, H. Cheng, T. M. Roberts, and J. J. Zhao, "Targeting the phosphoinositide 3-kinase pathway in cancer," Nature Reviews Drug Discovery, vol. 8, no. 8, pp. 627-644, 2009.

[37] T. L. Yuan and L. C. Cantley, "PI3K pathway alterations in cancer: variations on a theme," Oncogene, vol. 27, no. 41, pp. 5497-5510, 2008.

[38] M. P. Myers, I. Pass, I. H. Batty et al., "The lipid phosphatase activity of PTEN is critical for its tumor supressor function," Proceedings of the National Academy of Sciences, vol. 95, no. 23, pp. 13513-13518, 1998.

[39] I. U. Agoulnik, M. C. Hodgson, W. A. Bowden, and M. M. Ittmann, "INPP4B: the new kid on the PI3K block," Oncotarget, vol. 2, no. 4, pp. 321-328, 2011.

[40] A. M. Ochnik and R. C. Baxter, "Combination therapy approaches to target insulin-like growth factor receptor signaling in breast cancer," Endocrine-Related Cancer, vol. 23, no. 11, pp. R527-R550, 2016.

[41] Y. Samuels, Z. Wang, A. Bardelli et al., "High frequency of mutations of the PIK3CA gene in human cancers," Science, vol. 304, no. 5670, p. 554, 2004.

[42] C.-H. Huang, D. Mandelker, O. Schmidt-Kittler et al., "The structure of a human $\mathrm{p} 110 \alpha / \mathrm{p} 85 \alpha$ complex elucidates the effects of oncogenic PI3Kalpha mutations," Science, vol. 318, no. 5857, pp. 1744-1748, 2007.

[43] Y. Hao, C. Wang, B. Cao et al., "Gain of interaction with IRS1 by $110 \alpha$-helical domain mutants is crucial for their oncogenic functions," Cancer Cell, vol. 23, no. 5, pp. 583-593, 2013.

[44] N. M. Keegan, J. P. Gleeson, B. T. Hennessy, and P. G. Morris, "PI3K inhibition to overcome endocrine resistance in breast cancer," Expert Opinion on Investigational Drugs, vol. 27, no. 1, pp. 1-15, 2018.

[45] S.-M. Maira, S. Pecchi, A. Huang et al., "Identification and characterization of NVP-BKM120, an orally available panclass I PI3-kinase inhibitor," Molecular Cancer Therapeutics, vol. 11, no. 2, pp. 317-328, 2012.

[46] C. Jin, A. Wang, J. Chen, X. Liu, and G. Wang, "Relationship between expression and prognostic ability of PTEN, STAT3 and VEGF-C in colorectal cancer," Experimental and Therapeutic Medicine, vol. 4, no. 4, pp. 633-639, 2012.

[47] A. Krohn, T. Diedler, L. Burkhardt et al., "Genomic deletion of PTEN is associated with tumor progression and early PSA recurrence in ERG fusion-positive and fusion-negative prostate cancer," The American Journal of Pathology, vol. 181, no. 2, pp. 401-412, 2012.

[48] J. Chen, T. Li, Q. Liu et al., "Clinical and prognostic significance of HIF- $1 \alpha$, PTEN, CD44v6, and survivin for gastric cancer: a meta-analysis," PLoS One, vol. 9, no. 3, Article ID e91842, 2014.

[49] Y. Lu, Y.-Z. Lin, R. LaPushin et al., "The PTEN/MMAC1/ TEP tumor suppressor gene decreases cell growth and induces apoptosis and anoikis in breast cancer cells," Oncogene, vol. 18, no. 50, pp. 7034-7045, 1999.

[50] S. Li, Y. Shen, M. Wang et al., "Loss of PTEN expression in breast cancer: association with clinicopathological characteristics and prognosis," Oncotarget, vol. 8, no. 19, pp. 32043-32054, 2017.

[51] N. Burrows, M. Babur, J. Resch et al., "GDC-0941 inhibits metastatic characteristics of thyroid carcinomas by targeting both the phosphoinositide-3 kinase (PI3K) and hypoxiainducible factor-1- $\alpha$ (HIF-1- $\alpha$ ) pathways," The Journal of Clinical Endocrinology \& Metabolism, vol. 96, no. 12, pp. E1934-E1943, 2011.
[52] Z. Q. Zou, L. N. Zhang, F. Wang, J. Bellenger, Y. Z. Shen, and X. H. Zhang, "The novel dual PI3K/mTOR inhibitor GDC0941 synergizes with the MEK inhibitor U0126 in non-small cell lung cancer cells," Molecular Medicine Reports, vol. 5, no. 5, pp. 503-508, 2012.

[53] P. Schmid, S. E. Pinder, D. Wheatley et al., "Phase II randomized preoperative window-of-opportunity study of the PI3K inhibitor pictilisib plus anastrozole compared with anastrozole alone in patients with estrogen receptor-positive breast cancer," Journal of Clinical Oncology, vol. 34, no. 17, pp. 1987-1994, 2016.

[54] I. E. Krop, I. A. Mayer, V. Ganju et al., "Pictilisib for oestrogen receptor-positive, aromatase inhibitor-resistant, advanced or metastatic breast cancer (FERGI): a randomised, double-blind, placebo-controlled, phase 2 trial," The Lancet Oncology, vol. 17, no. 6, pp. 811-821, 2016.

[55] J. Rodon, J. Bendell, R. A. Abdul et al., "P3-16-01: safety profile and clinical activity of single-agent BKM120, a panclass I PI3K inhibitor, for the treatment of patients with metastatic breast carcinoma," Cancer Research, vol. 71, no. 24, 2011.

[56] Y. Ando, M. Inada-Inoue, A. Mitsuma et al., "Phase I doseescalation study of buparlisib (BKM120), an oral pan-class I PI3K inhibitor, in Japanese patients with advanced solid tumors," Cancer Science, vol. 105, no. 3, pp. 347-353, 2014.

[57] C. X. Ma, C. Sanchez, F. Gao et al., "A phase I study of the AKT inhibitor MK-2206 in combination with hormonal therapy in postmenopausal women with estrogen receptorpositive metastatic breast cancer," Clinical Cancer Research, vol. 22, no. 11, pp. 2650-2658, 2016.

[58] S. Loibl, L. de la Pena, V. Nekljudova et al., "Neoadjuvant buparlisib plus trastuzumab and paclitaxel for women with HER2+ primary breast cancer: a randomised, double-blind, placebo-controlled phase II trial (NeoPHOEBE)," European Journal of Cancer, vol. 85, pp. 133-145, 2017.

[59] J. Baselga, S.-A. Im, H. Iwata et al., "Buparlisib plus fulvestrant versus placebo plus fulvestrant in postmenopausal, hormone receptor-positive, HER2-negative, advanced breast cancer (BELLE-2): a randomised, double-blind, placebocontrolled, phase 3 trial," The Lancet Oncology, vol. 18, no. 7, pp. 904-916, 2017.

[60] A. Di Leo, S. Johnston, K. S. Lee et al., "Buparlisib plus fulvestrant in postmenopausal women with hormone-receptor-positive, HER2-negative, advanced breast cancer progressing on or after mTOR inhibition (BELLE-3): a randomised, double-blind, placebo-controlled, phase 3 trial," The Lancet Oncology, vol. 19, no. 1, pp. 87-100, 2018.

[61] S. Jain, C. A. Santa-Maria, A. Rademaker, F. J. Giles, M. Cristofanilli, and W. J. Gradishar, "Phase I study of alpelisib (BYL-719) and T-DM1 in HER2-positive metastatic breast cancer after trastuzumab and taxane therapy," Journal of Clinical Oncology, vol. 35, no. 15_suppl, p. 1026, 2017.

[62] M. J. Ellis, L. Lin, R. Crowder et al., "Phosphatidyl-inositol-3kinase alpha catalytic subunit mutation and response to neoadjuvant endocrine therapy for estrogen receptor positive breast cancer," Breast Cancer Research and Treatment, vol. 119, no. 2, pp. 379-390, 2010.

[63] T. W. Miller, J. M. Balko, and C. L. Arteaga, "Phosphatidylinositol 3-kinase and antiestrogen resistance in breast cancer," Journal of Clinical Oncology, vol. 29, no. 33, pp. 4452-4461, 2011.

[64] C. Fritsch, A. Huang, C. Chatenay-Rivauday et al., "Characterization of the novel and specific PI3K $\alpha$ inhibitor NVPBYL719 and development of the patient stratification 
strategy for clinical trials," Molecular Cancer Therapeutics, vol. 13, no. 5, pp. 1117-1129, 2014.

[65] F. Morgillo, C. M. Della Corte, A. Diana et al., "Phosphatidylinositol 3-kinase (PI3K $\alpha$ )/AKT axis blockade with taselisib or ipatasertib enhances the efficacy of anti-microtubule drugs in human breast cancer cells," Oncotarget, vol. 8, no. 44, pp. 76479-76491, 2017.

[66] American Association for Cancer Research, "Alpelisib extends PFS in PIK3CA-mutant breast cancer," Cancer Discovery, vol. 9, no. 1, pp. 6-7, 2019.

[67] A. Ardestani, B. Lupse, Y. Kido, G. Leibowitz, and K. Maedler, "mTORC1 signaling: a double-edged sword in diabetic $\beta$ cells," Cell Metabolism, vol. 27, no. 2, pp. 314-331, 2018.

[68] Y. Ji, W. Di, Q. Yang, Z. Lu, W. Cai, and J. Wu, "Inhibition of autophagy increases proliferation inhibition and apoptosis induced by the PI3K/mTOR inhibitor NVP-BEZ235 in breast cancer cells," Clinical Laboratory, vol. 61, pp. 10431051, 2015.

[69] J. Rodon, A. Pérez-Fidalgo, I. E. Krop et al., "Phase 1/1b dose escalation and expansion study of BEZ235, a dual PI3K/ mTOR inhibitor, in patients with advanced solid tumors including patients with advanced breast cancer," Cancer Chemotherapy and Pharmacology, vol. 82, no. 2, pp. 285-298, 2018.

[70] J. Yang, J. Nie, X. Ma, Y. Wei, Y. Peng, and X. Wei, “Targeting PI3K in cancer: mechanisms and advances in clinical trials," Molecular Cancer, vol. 18, no. 1, p. 26, 2019.

[71] G.-M. Karthik, R. Ma, J. Lövrot et al., "mTOR inhibitors counteract tamoxifen-induced activation of breast cancer stem cells," Cancer Letters, vol. 367, no. 1, pp. 76-87, 2015.

[72] P. De, Y. Sun, J. H. Carlson, L. S. Friedman, B. R. LeylandJones, and N. Dey, "Doubling down on the PI3K-AKTmTOR pathway enhances the antitumor efficacy of PARP inhibitor in triple negative breast cancer model beyond BRCA-ness," Neoplasia, vol. 16, no. 1, pp. 43-72, 2014.

[73] R. Ali, E. A. Rakha, S. Madhusudan, and H. E. Bryant, "DNA damage repair in breast cancer and its therapeutic implications," Pathology, vol. 49, no. 2, pp. 156-165, 2017.

[74] J. Á. F. Vara, E. Casado, J. de Castro, P. Cejas, C. BeldaIniesta, and M. González-Barón, "PI3K/AKT signaling pathway and cancer," Cancer Treatment Reviews, vol. 30, no. 2, pp. 193-204, 2004.

[75] A. Toker and L. C. Cantley, "Signalling through the lipid products of phosphoinositide-3-OH kinase," Nature, vol. 387, no. 6634, pp. 673-676, 1997.

[76] D. D. Sarbassov, D. A. Guertin, S. M. Ali, and D. M. Sabatini, "Phosphorylation and regulation of Akt/PKB by the rictormTOR complex," Science, vol. 307, no. 5712, pp. 1098-1101, 2005.

[77] S. R. Datta, A. Brunet, and M. E. Greenberg, "Cellular survival: a play in three Akts," Genes \& Development, vol. 13, no. 22, pp. 2905-2927, 1999.

[78] B. D. Manning and A. Toker, "AKT/PKB signaling: navigating the network," Cell, vol. 169, no. 3, pp. 381-405, 2017.

[79] P. S. Mundi, J. Sachdev, C. McCourt, and K. Kalinsky, "AKT in cancer: new molecular insights and advances in drug development," British Journal of Clinical Pharmacology, vol. 82, no. 4, pp. 943-956, 2016.

[80] J. A. McCubrey, L. S. Steelman, F. E. Bertrand et al., "GSK-3 as potential target for therapeutic intervention in cancer," Oncotarget, vol. 5, no. 10, pp. 2881-2911, 2014.

[81] J. Dong, J. Peng, H. Zhang et al., "Role of glycogen synthase kinase $3 \beta$ in rapamycin-mediated cell cycle regulation and chemosensitivity," Cancer Research, vol. 65, no. 5, pp. 1961-1972, 2005.

[82] M. Farago, I. Dominguez, E. Landesman-Bollag et al., "Kinase-inactive glycogen synthase kinase $3 \beta$ promotes Wnt signaling and mammary tumorigenesis," Cancer Research, vol. 65, no. 13, pp. 5792-5801, 2005.

[83] G. Tzivion, M. Dobson, and G. Ramakrishnan, "FoxO transcription factors: regulation by AKT and 14-3-3 proteins," Biochimica et Biophysica Acta (BBA)-Molecular Cell Research, vol. 1813, no. 11, pp. 1938-1945, 2011.

[84] K. Du and M. Montminy, "CREB is a regulatory target for the protein kinase AKT/PKB," Journal of Biological Chemistry, vol. 273, no. 49, pp. 32377-32379, 1998.

[85] A. R. Hussain, S. O. Ahmed, M. Ahmed et al., "Cross-talk between NFkB and the PI3-kinase/AKT pathway can be targeted in primary effusion lymphoma (PEL) cell lines for efficient apoptosis," PLoS One, vol. 7, no. 6, Article ID e39945, 2012.

[86] G. Pérez-Tenorio and O. Stål, "Activation of AKT/PKB in breast cancer predicts a worse outcome among endocrine treated patients," British Journal of Cancer, vol. 86, no. 4, pp. 540-545, 2002.

[87] E. Tokunaga, Y. Kimura, K. Mashino et al., "Activation of $\mathrm{PI} 3 \mathrm{~K} /$ Akt signaling and hormone resistance in breast cancer," Breast Cancer, vol. 13, no. 2, pp. 137-144, 2006.

[88] J. D. Carpten, A. L. Faber, C. Horn et al., “A transforming mutation in the pleckstrin homology domain of AKT1 in cancer," Nature, vol. 448, no. 7152, pp. 439-444, 2007.

[89] D. M. Hyman, L. Smyth, P. L. Bedard et al., "Abstract B109: AZD5363, a catalytic pan-Akt inhibitor, in Akt1 E17K mutation positive advanced solid tumors," Molecular Cancer Therapeutics, vol. 14, no. 12 Supplement 2, p. B109, 2015.

[90] H. Hua, Q. Kong, H. Zhang, J. Wang, T. Luo, and Y. Jiang, "Targeting mTOR for cancer therapy," Journal of Hematology \& Oncology, vol. 12, no. 1, p. 71, 2019.

[91] Y. Rabanal-Ruiz, E. G. Otten, and V. I. Korolchuk, "mTORC1 as the main gateway to autophagy," Essays in Biochemistry, vol. 61, no. 6, pp. 565-584, 2017.

[92] C. J. Potter, L. G. Pedraza, and T. Xu, "Akt regulates growth by directly phosphorylating Tsc2," Nature Cell Biology, vol. 4, no. 9, pp. 658-665, 2002.

[93] R. A. Saxton and D. M. Sabatini, "mTOR signaling in growth, metabolism, and disease," Cell, vol. 168, no. 6, pp. 960-976, 2017.

[94] E. Jacinto, R. Loewith, A. Schmidt et al., "Mammalian TOR complex 2 controls the actin cytoskeleton and is rapamycin insensitive," Nature Cell Biology, vol. 6, no. 11, pp. 11221128, 2004.

[95] M. Laplante and D. M. Sabatini, "mTOR signaling in growth control and disease," Cell, vol. 149, no. 2, pp. 274-293, 2012.

[96] L. R. Pearce, D. Komander, and D. R. Alessi, "The nuts and bolts of AGC protein kinases," Nature Reviews Molecular Cell Biology, vol. 11, no. 1, pp. 9-22, 2010.

[97] M. M. Morrison, C. D. Young, S. Wang et al., "mTOR directs breast morphogenesis through the PKC-alpha-Rac1 signaling Axis," PLoS Genetics, vol. 11, no. 7, Article ID e1005291, 2015.

[98] M. A. Bruhn, R. B. Pearson, R. D. Hannan, and K. E. Sheppard, "Second AKT: the rise of SGK in cancer signalling," Growth Factors, vol. 28, no. 6, pp. 394-408, 2010.

[99] M. Jhanwar-Uniyal, A. G. Amin, J. B. Cooper, K. Das, M. H. Schmidt, and R. Murali, "Discrete signaling mechanisms of mTORC1 and mTORC2: connected yet apart in 
cellular and molecular aspects," Advances in Biological Regulation, vol. 64, pp. 39-48, 2017.

[100] R. Vadla and D. Haldar, "Mammalian target of rapamycin complex 2 (mTORC2) controls glycolytic gene expression by regulating Histone H3 Lysine 56 acetylation," Cell Cycle, vol. 17, no. 1, pp. 110-123, 2018.

[101] E. J. Macaskill, J. M. S. Bartlett, V. S. Sabine et al., "The mammalian target of rapamycin inhibitor everolimus (RAD001) in early breast cancer: results of a pre-operative study," Breast Cancer Research and Treatment, vol. 128, no. 3, pp. 725-734, 2011.

[102] L. Du, X. Li, L. Zhen et al., "Everolimus inhibits breast cancer cell growth through PI3K/AKT/mTOR signaling pathway," Molecular Medicine Reports, vol. 17, no. 5, pp. 7163-7169, 2018.

[103] M. I. Shtivelband, "Everolimus in hormone receptor-positive advanced breast cancer: targeting receptor-based mechanisms of resistance," The Breast, vol. 22, no. 4, pp. 405-410, 2013.

[104] E. Y. Leung, M. Askarian-Amiri, G. J. Finlay, G. W. Rewcastle, and B. C. Baguley, "Potentiation of growth inhibitory responses of the mTOR inhibitor everolimus by dual mTORC1/2 inhibitors in cultured breast cancer cell lines," PLoS One, vol. 10, no. 7, Article ID e0131400, 2015.

[105] Y. Gökmen-Polar, Y. Liu, R. A. Toroni et al., "Investigational drug MLN0128, a novel TORC1/2 inhibitor, demonstrates potent oral antitumor activity in human breast cancer xenograft models," Breast Cancer Research and Treatment, vol. 136, no. 3, pp. 673-682, 2012.

[106] B. Basu, E. Dean, M. Puglisi et al., "First-in-human pharmacokinetic and pharmacodynamic study of the dual m-TORC 1/2 inhibitor AZD2014," Clinical Cancer Research, vol. 21, no. 15, pp. 3412-3419, 2015.

[107] S. M. Guichard, Z. Howard, D. Heathcote et al., “Abstract 917: AZD2014, a dual mTORC1 and mTORC2 inhibitor is differentiated from allosteric inhibitors of mTORC1 in ER+ breast cancer," Cancer Research, vol. 72, no. 8 Supplement, p. 917, 2012.

[108] V. S. Rodrik-Outmezguine, M. Okaniwa, Z. Yao et al., "Overcoming mTOR resistance mutations with a newgeneration mTOR inhibitor," Nature, vol. 534, no. 7606, pp. 272-276, 2016.

[109] T. M. A. Abdel-Fatah, A. Arora, P. Moseley et al., "ATM, ATR and DNA-PKcs expressions correlate to adverse clinical outcomes in epithelial ovarian cancers," BBA Clinical, vol. 2, pp. 10-17, 2014.

[110] T. Tsuji, L. M. Sapinoso, T. Tran et al., "CC-115, a dual inhibitor of mTOR kinase and DNA-PK, blocks DNA damage repair pathways and selectively inhibits ATM-deficient cell growth in vitro," Oncotarget, vol. 8, no. 43, pp. 74688-74702, 2017.

[111] W. M. Ciszewski, M. Tavecchio, J. Dastych, and N. J. Curtin, "DNA-PK inhibition by NU7441 sensitizes breast cancer cells to ionizing radiation and doxorubicin," Breast Cancer Research and Treatment, vol. 143, no. 1, pp. 47-55, 2014.

[112] T. A. Werfel, S. Wang, M. A. Jackson et al., "Selective mTORC2 inhibitor therapeutically blocks breast cancer cell growth and survival," Cancer Research, vol. 78, no. 7, pp. 1845-1858, 2018.

[113] K. Mardilovich, S. L. Pankratz, and L. M. Shaw, "Expression and function of the insulin receptor substrate proteins in cancer," Cell Communication and Signaling, vol. 7, no. 1, p. 14, 2009.
[114] C. M. Taniguchi, B. Emanuelli, and C. R. Kahn, "Critical nodes in signalling pathways: insights into insulin action," Nature Reviews Molecular Cell Biology, vol. 7, no. 2, pp. 85-96, 2006.

[115] M. Björnholm, A. R. He, A. Attersand et al., "Absence of functional insulin receptor substrate-3 (IRS-3) gene in humans," Diabetologia, vol. 45, no. 12, pp. 1697-1702, 2002.

[116] D. Cai, S. Dhe-Paganon, P. A. Melendez, J. Lee, and S. E. Shoelson, "Two new substrates in insulin signaling, IRS5/DOK4 and IRS6/DOK5," Journal of Biological Chemistry, vol. 278, no. 28, pp. 25323-25330, 2003.

[117] K. Karrman, M. Isaksson, K. Paulsson, and B. Johansson, "The insulin receptor substrate 4 gene (IRS4) is mutated in paediatric T-cell acute lymphoblastic leukaemia," British Journal of Haematology, vol. 155, no. 4, pp. 516-519, 2011.

[118] J. Weischenfeldt, T. Dubash, A. P. Drainas et al., "Pan-cancer analysis of somatic copy-number alterations implicates IRS4 and IGF2 in enhancer hijacking," Nature Genetics, vol. 49, no. 1, pp. 65-74, 2017.

[119] M. F. White, "The IRS-signalling system: a network of docking proteins that mediate insulin action," Insulin Action, vol. 182, pp. 3-11, 1998.

[120] G. J. Ikink, M. Boer, E. R. M. Bakker, and J. Hilkens, "IRS4 induces mammary tumorigenesis and confers resistance to HER2-targeted therapy through constitutive PI3K/AKTpathway hyperactivation," Nature Communications, vol. 7, no. 1, p. 13567, 2016.

[121] G. Hoxhaj, K. Dissanayake, and C. MacKintosh, "Effect of IRS4 levels on PI 3-kinase signalling," PLoS One, vol. 8, no. 9, Article ID e73327, 2013.

[122] K. Nash, W. Chen, M. Salganik, and N. Muzyczka, "Identification of cellular proteins that interact with the adenoassociated virus rep protein," Journal of Virology, vol. 83, no. 1, pp. 454-469, 2009.

[123] H. Qiu, F. Zappacosta, W. Su, R. S. Annan, and W. T. Miller, "Interaction between Brk kinase and insulin receptor substrate-4," Oncogene, vol. 24, no. 36, pp. 5656-5664, 2005.

[124] J. Jiang, F. Gui, Z. He et al., "Targeting BRK-positive breast cancers with small-molecule kinase inhibitors," Cancer Research, vol. 77, no. 1, pp. 175-186, 2017.

[125] K. Berns, H. M. Horlings, B. T. Hennessy et al., "A functional genetic approach identifies the PI3K pathway as a major determinant of trastuzumab resistance in breast cancer," Cancer Cell, vol. 12, no. 4, pp. 395-402, 2007.

[126] P. J. A. Eichhorn, M. Gili, M. Scaltriti et al., "PI3K hyperactivation results in lapatinib resistance that is reversed by the mTOR/PI3K inhibitor NVP-BEZ235," Cancer Research, vol. 68, no. 22, pp. 9221-9230, 2008. 